\title{
Long non-coding RNA ATB promotes glioma malignancy by negatively regulating miR-200a
}

\author{
Chun-Chun Ma ${ }^{1,2+}$, Zhang Xiong ${ }^{1,2+}$, Guan-Nan Zhu ${ }^{1,2}$, Chao Wang ${ }^{1,2}$, Gang Zong ${ }^{1,2}$, Hong-Liang Wang ${ }^{1,2}$, \\ Er-Bao Bian ${ }^{1,2}$ and Bing Zhao ${ }^{1,2^{*}}$
}

\begin{abstract}
Background: Glioma is one of the most common and aggressive primary malignant tumor in the brain. Accumulating evidences indicated that aberrantly expressed non-coding RNAs (ncRNAs), including long non-coding RNAs (IncRNAs) and microRNAs (miRNAs), contribute to tumorigenesis. However, potential mechanisms between IncRNAs and miRNAs in glioma remain largely unknown.
\end{abstract}

Methods: Long non-coding RNA activated by TGF- $\beta$ (LnCRNA-ATB) expression in glioma tissues and cells was quantified by quantitative reverse transcription-PCR. Glioma cell lines U251 and A172 were transfected with sh-ATB, miR-200a mimics, miR-200a inhibitors, after we assayed the cell phenotype and expression of the relevant molecules. Dual-luciferase reporter assay, RIP and a xenograft mouse model were used to examine the expression of sh-ATB and its target gene miR-200a.

Results: ATB is abnormally up-regulated both in glioma tissues and cell lines compared with normal brain tissues, and glioma patients with high ATB expression had shorter overall survival time. Knockdown of ATB significantly inhibits glioma malignancy, including cell proliferation, colony formation, migration, invasion in vitro, and the xenograft tumor formation in vivo. In addition, ATB was confirmed to target miR-200a, and miR-200a inhibition reversed the malignant characteristics of ATB knockdown on glioma cells. In particular, ATB may act as a ceRNA, effectively becoming a sink for miR-200a, thereby modulating the derepression of TGF- $\beta 2$.

Conclusions: Our findings suggest that ATB plays an oncogenic role of glioma cells by inhibiting miR-200a and facilitating TGF- $\beta 2$ in glioma, thereby may represent a potential therapeutic target for the treatment of human glioma.

Keywords: Glioma, IncRNA, ATB, miRNA, miR-200a, ceRNA, TGF- $\beta 2$

\section{Background}

Glioma poses a tremendous threat to public health with an incidence of $\sim 5$ cases per 100,000 persons and its mortality is high around the world. Glioma is categorized into four histopathologic grades (I-IV) based on the degree of malignancy according to World Health Organization (WHO) classification [1]. The majority patients suffered from glioma are diagnosed at the

\footnotetext{
*Correspondence: aydzhb@126.com

${ }^{\dagger}$ Equal contributors

'Department of Neurosurgery, The Second Affiliated Hospital of Anhui Medical University, 678 Fu Rong Road, Hefei, Anhui Province, China 230601 ${ }^{2}$ Cerebral Vascular Disease Research Center, Anhui Medical University, $678 \mathrm{Fu}$ Rong Road, Hefei, Anhui Province, China 230601
}

advanced stages and exhibit extremely poor prognosis. Combined with maximal safe surgical resection, standard radiation therapy and chemotherapy, the most advanced treatment is formed, while the most malignant glioma called gliobastoma multiform is lined with an average life expectancy of only 14 months [2]. Fortunately, significant progress has been made in understanding the molecular mechanisms of glioma. Though clinical application is largely palliative, the fatality rate remains high in glioma patients. Therefore, it is extremely vital for us to elucidate new mechanisms associated with glioma development and establish potential therapeutic targets for the treatment of human glioma. 
Just as the human genome project which delineated that only a small amount of mammalian genome is encoded proteins while the vast majority of mammalian genome are transcribed as non-coding RNAs (ncRNAs), such as long noncoding RNAs(lncRNAs) [3, 4]. LncRNAs are a class of transcripts longer than 200 nucleotides in length with little functional protein-coding ability. Recently, many studies have demonstrated that IncRNAs could regulate gene expression at multiple levels, including transcriptional, post-transcriptional and epigenetic modulation [5-7]. Numerous studies also indicated that dysregulated lncRNAs are involved in the biological process during cancer development and progression $[8,9]$ Examples like lncRNAs include HOTAIR (HOX transcript antisense intergenic RNA) [10], CRNDE(Colorectal neoplasia differentially expressed) [11], MEG3 (Maternally Expressed Gene 3) [12]. Yet, the biological roles of lncRNAs in glioma are largely unknown. Long noncoding RNA activated by TGF- $\beta$ (lncRNA-ATB) was initially identified as a lncRNA in Hepatocellular carcinoma (HCC) and its expression was significantly up-regulated both in HCC cells and HCC samples [13]. Furthermore, ATB was also overexpressed in other cancers, including breast cancer [14] and colorectal cancer [15]. Therefore, the aberrant expression level of ATB was involved in a series of cancer progression. However, functional mechanism and potential biological role of ATB in human glioma are still unknown.

MiRNAs are highly conserved among species, and play important roles in a variety of biological and pathological processes. A number of studies indicated that deviant expression of miRNAs contributes to tumorigenesis and plays a critical role in regulating the biological behaviors of tumor cells by modulating the protein or mRNA levels of its downstream target genes $[16,17]$. Dysregulation of miRNAs in glioma has also been reported, and certain miRNAs have been functionally involved in glioma. Previous studies have demonstrated that miR-200a as a member of the miR-200 family, which exerts as a tumor-suppressor gene and is down-regulated in many tumors, including glioma [18]. However, the molecular mechanism of miR-200a deregulation and how such deregulation contributes to glioma tumorigenesis remains abstruse.

In the present study, we aimed to investigate the functional expression and clinical significance of ATB in human glioma. ATB was significantly up-regulated both in glioma tissues and cell lines, whereas knockdown of ATB diminished cell proliferation, migration and invasion in glioma. In addition, the interaction among ATB, miR-200a and TGF- $\beta 2$ was also studied in order to reveal the underlying mechanisms. We identified that ATB may act as a ceRNA of miR-200a, which resulted in the derepression of TGF- $\beta 2$. These findings will give a novel strategy for the treatment of glioma.

\section{Methods}

\section{Human tissue samples}

Seventy-nine glioma tissues and 15 normal brain tissues (NBTs) were obtained from the Department of Neurosurgery, the Second Affiliated Hospital of AnHui Medical University during 2011-2014. These glioma samples were from 50 males and 29 females with age ranging from 13 to 73 years (median, 47 years). All samples had confirmed pathological diagnosis and were divided into low grade (grade I-II) and high grade (grade III-IV) according to the WHO classification by neuropathologists. Informed consents were obtained from all patients, and this study was approved by the Clinical Research Ethics Committee at the Second Affiliated Hospital of AnHui Medical University.

\section{Cell culture}

The human glioma cell lines (U251 and A172) were purchased from the Chinese Academy of Sciences (Shanghai, China) and cultivated in Dulbecco's modified Eagle's medium (DMEM; Hyclone, Logan, UT, USA) with high glucose supplemented with $10 \%$ fetal bovine serum (FBS, Gibco, Carlsbad, CA, USA) and streptomycin $(100 \mu \mathrm{g} / \mathrm{ml})$, penicillin $(100 \mathrm{U} / \mathrm{ml})$. All cell lines were cultured at $37{ }^{\circ} \mathrm{C}$ in a humidified incubator with $5 \% \mathrm{CO}_{2}$.

\section{Cell transfection}

Short-hairpin RNA plasmid directed knock-down human ATBs (GenePharma, Shanghai, China), and was indicated as sh-ATB (sh-ATB sense:5'-GATCCGCCTGTCTGT ATTTGCGAATACCTTTTTCAAGAGAAAAGGTATTC GCAAATACAGACAGGCTTTTTTG-3' and anti-sense: 5'-AATTCAAAAAAGCCTGTCTGTATTTGCGAATAC CTTTTCTCTTGAAAAAGGTATTCGCAAATACAGAC AGGCG-3') and its corresponding non-targeting sequence (sh-control) (sh-control sense: 5'-GATCCGTTCTCCGA ACGTGTCACGTTTCAAGAGAACGTGACACGTTCG GAGAACTTTTTTG-3' and anti-sense: 5'-AATTCA AAAAAGTTCTCCGAACGTGTCACGTTCTCTTGA AACGTGACACGTTCGGAGAACG-3') plasmid (GenePharma, Shanghai, China) were transfected into U251 and A172 cells respectively by using Lipofectamine2000 (Invitrogen, USA) according to the manufacturer's protocol. MiR-200a mimics, miR-200a inhibitors and miR-200a negative control (NC) were obtained from RiboBio (RiboBio, Guangzhou, China), and transfected into cell lines as the above described.

\section{RNA extraction and quantitative Real-time PCR}

Total RNAs were extracted from tissues and cultured cells using Trizol reagent (Invitrogen, USA) according to the manufacturer's protocol. Using a Nanodrop Spectrophotometer (IMPLEN GmbH, Munich, Germany), RNA concentration and quality were determined by the 260/ $280 \mathrm{~nm}$ absorbance. Then RNA was reversely transcribed 
into High Capacity cDNA by using PrimeScript ${ }^{\mathrm{TM}}$ RT Master Mix (Perfect Real Time) (TaKaRa Biotechnology, Dalian, China). Maxima SYBR Green/ROX qPCR Master Mix (Thermo Fisher Scientific, USA) was used for quantitative Real-time PCR. The primers for genes were determined as follows: ATB forward 5'-ACAAGCTGTGCAGTCTCAGG-3', reverse 5' - CTAGGCCCAAAGACAATGGA-3'; TGF$\beta 2$ forward 5'-CACCATAAAGACAGGAACCTG-3', reverse 5' -GGAGGTGCCATCAATACCTGC-3'; GAPDH forward 5'-AGCAAGAGCACAAGAGGAAG-3', reverse 5'-GGTTGAGCACAGGGTACTTT-3'. All-in-One ${ }^{\text {тм }}$ miRNA First-Strand cDNA Synthesis Kit (Genecopoeia, Guangzhou, China) was used for miRNA reverse transcription and RT-QPCR was conducted using All-in-One ${ }^{\text {тм }}$ miRNA qPCR Kit (Genecopoeia, Guangzhou, China) of miR-200a and U6 (miR-200a (Cat\#HmiRQP0298) and U6 (Cat\#HmiRQP9001), Genecopoeia, Guangzhou, China), respectively, by using the ABI 7100 (Applied Biosystems, Darmstadt, Germany). GAPDH and U6 were used as internal controls for ATB, TGF- $\beta 2$ and miR-200a. PCR Cycling conditions for mRNA was $2 \mathrm{~min}$ at $50{ }^{\circ} \mathrm{C}, 10 \mathrm{~min}$ at $95^{\circ} \mathrm{C}$ and followed by 45 cycles of $95^{\circ} \mathrm{C}$ for $15 \mathrm{~s}, 60{ }^{\circ} \mathrm{C}$ for 60s; for miRNA was $10 \mathrm{~min}$ at $95{ }^{\circ} \mathrm{C}$ and followed by 45 cycles of $95^{\circ} \mathrm{C}$ for 10 s, $60{ }^{\circ} \mathrm{C}$ for 20 s and $72{ }^{\circ} \mathrm{C}$ for $12 \mathrm{~s}$. All qRT-PCR reactions were performed in triplicate. Relative quantification of gene expression was calculated by the $2^{-\Delta \Delta \mathrm{Ct}}$ method.

\section{Cell proliferation assay}

Cell Counting Kit-8 (CCK8, Beyotime Institute of Biotechnology, Jiangsu, China) was used to detect the cell's proliferation ability. Cells were placed into 96-well plates at the density of 2000 cells/well. Approximately $20 \mu \mathrm{l}$ of CCK8 regent was added to each well after transfection, and incubated at $37{ }^{\circ} \mathrm{C}$ for $2 \mathrm{~h}$. Absorbance was measured at a wavelength of $450 \mathrm{~nm}$ by using a ST-360 micro-plate reader (KHB, Shanghai, China). Three replicate wells were set up in each group and experiments were repeated three times.

\section{Clony formation assay}

For the clone formation assay, approximately 200 viable cells were seeded in 6-well plates in triplicate and incubated at $37{ }^{\circ} \mathrm{C}$ with $10 \%$ fetal bovine serum. After 12 days, cells were fixed with $4 \%$ polyoxymethylene and stained with $1.5 \%$ methylene blue. Colony formation ratio was calculated as (number of cells/initiative cell $\times 100(\%)$ ).

\section{Wound healing assay}

Cells were cultured in 6-well plates. When $95 \%$ confluency was reached, cell layers were wounded using a $10 \mu \mathrm{L}$ tip to produce a gap, gently washed, and cultured with serum-free medium for $24 \mathrm{~h}$. The wounded gaps were photographed with a light microscope (IX71; Olympus, Tokyo, Japan) at $\times 200$ magnification. Lines were drawn along the leading edges of the cells, and the gap distances of migrating cells from five different areas for each wound were measured and analyzed.

\section{Cell invasion assays}

Cell invasion ability was tested by using 24-well chambers with $8 \mu \mathrm{m}$ pore size (Corning, USA). $8 \times 10^{4}$ cells were resuspended in $150 \mu \mathrm{l}$ serum-free medium and seeded into the upper chamber pre-coated with $500 \mathrm{ng} / \mathrm{ml}$ Matrigel solution (BD, USA) invasion assay, while $500 \mu \mathrm{l}$ of $10 \%$ FBS medium was placed in the lower chamber, After incubation at $37{ }^{\circ} \mathrm{C}$ for $48 \mathrm{~h}$ for invasion assay. Cells on the upper chamber membrane were scraped off by cotton swab. Cells on the lower chamber membrane were fixed with $4 \%$ polyoxymethylene and stained with $0.1 \%$ crystal violet. Five predetermined fields were counted under a microscope $(\times 200)$. All assays were performed in triplicate.

\section{Luciferase reporter assays}

ATB fragment containing the predicted miR-200a binding site, the putative sequences of the binding site then cloned into a pmirGlO Dual-luciferase miRNA Target Expression Vector (Promega, Madison, WI, USA) to form the reporter vector pmiRGLO-ATB-wild-type (ATB-WT). To mutate the putative binding site of miR200a in ATB gene, the sequence of putative binding site was replaced as indicated and was named as pmiRGLOATB-mutated-type (ATB-MUT). pmirGLO-ATB-WT or pmirGLO-ATB-MUT was cotransfected with miR-200a mimics or miR-200a NC into glioma cells by using Lipofectamie 2000 (Invitrogen, USA). After $48 \mathrm{~h}$ transfection for luciferase assay using a Dual-Luciferase Reporter Assay System (Promega, Madison, WI, USA) according to the manufacturer's protocol.

Similarly the 3'-UTR of TGF- $\beta 2$ containing the putative miR-200a binding site, the putative sequences of the binding site were cloned into a pmirGlo Dual-luciferase miRNA Target Expression Vector to form the reporter vector TGF- $\beta 2$-wild-type (TGF- $\beta 2-W T$ ) (GenePharma). To mutate the putative binding site of miR-200a in the 3 '-UTR-containing vector, the sequence of putative binding site was replaced as indicated and was named as TGF- $\beta 2$-mutated-type (TGF- $\beta 2-M U T)$. The transfection procedure and measurement of Luciferase activities were handled similarly as described above. All assays were independently performed in triplicate.

\section{RNA immunoprecipitation}

EZ-Magna RIP RNA-binding protein immunoprecipitation kit (Millipore, Billerica, MA, USA) was used in RNA immunoprecipitation (RIP). RIP was implemented 
to pull down endogenous miR-200a associated with ATB in glioma cell lines, and was performed following the manufacturer's protocol. U251 and A172 cells were lysed by RIP lysis buffer, $100 \mu \mathrm{L}$ of cell lysate was incubated with RIP immunoprecipitation buffer containing magnetic beads conjugated with human anti-Argonaute2 (Ago2) antibody (Millipore) and normal mouse IgG (Millipore) was indicated as negative control. Samples were incubated with Proteinase $\mathrm{K}$ buffer and then target RNA was extracted. Purified RNA was subjected to RTQPCR analysis for further study.

\section{Western blot analysis}

Total proteins were extracted from the cells using RIPA buffer with PMSF (Beyotime Institute of Biotechnology) on ice, subjected to SDS-PAGE and electrophoretically transferred to polyvinylidene difluoride (PVDF) membranes. Membranes were incubated in $5 \%$ nonfat milk dissolved in Tris-buffered saline (TBS) containing $0.1 \%$ Tween-20 for $1.5 \mathrm{~h}$ at room temperature and then incubated with primary antibodies as follows: TGF- $\beta 2$ (1:1000, Abcam, EUGENE, USA), $\beta$-actin (1:1000, Santa Cruz Biotechnology). After incubation with secondary antibodies (Goat anti-rabbit or Goat anti-mouse, 1:5000 respectively; ZSGB-BIO, Beijing, China), immune complexes were visualized by SuperSignal ${ }^{\circ}$ West Femto Trial Kit (Thermo Fisher, USA) and blot bands were scanned using Find-do $\times 6$ Tanon (Tanon, Shanghai, China).

\section{Tumor xenograft formation assay in nude mice}

Four-week-old female BALB/C athymic nude mice were purchased from the National Laboratory Animal Center (Beijing, China). Experiments with nude mice were conducted strictly in accordance with a protocol approved by the Administrative Panel on Laboratory Animal Care of the Second Affiliated Hospital of AnHui Medical University. The animals were free to autoclaved food and water during the study. U251 cells transfected with sh-control or sh-ATB were collected, and $3 \times 10^{6}$ cells were subcutaneously injected into the left flank of the nude mice. Tumor volumes were examined every 5 days, and after 40 days, the mice were killed and tumor tissues were excised and weighed for further study.

\section{Immunohistochemistry}

Tumor tissues from subcutaneous implantation assay were fixed in $4 \%$ paraformaldehyde, and then were dehydrated, embedded in paraffin, and cut. Consecutive $4 \mu \mathrm{m}$ thick sections were analyzed by immunohistochemistry using antibodies against Ki-67 (Santa Cruz Biotechnology, USA).

\section{Statistical analysis}

Experimental data were presented as means \pm standard deviation (SD). GraphPad Prism V5.0 (GraphPad Software, Inc., La Jolla, CA, USA) software was used for statistical analysis. Differences were analyzed by SPSS 17.0 statistical software with the Student's t-test or one-way ANOVA. The
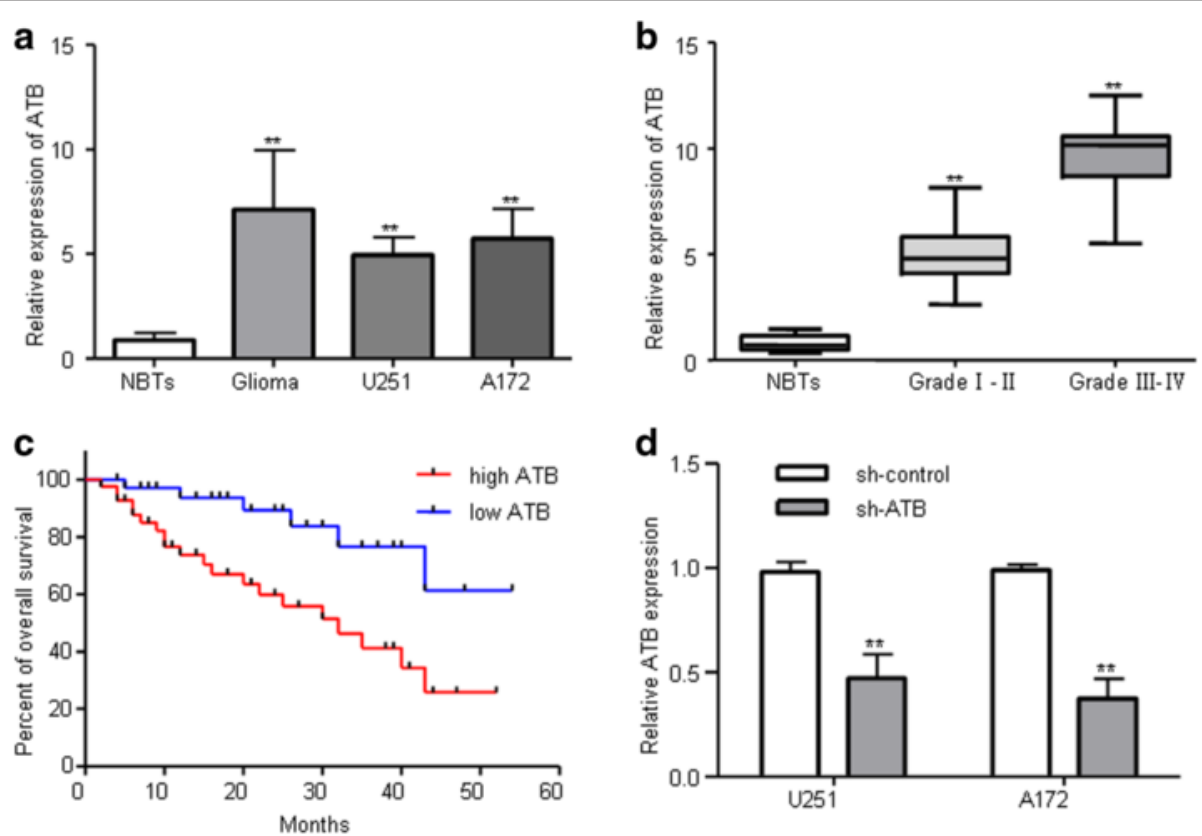

Fig. 1 ATB was highly expressed in human glioma. a RT-QPCR analysis of ATB expression in normal brain tissues (NBTs) ( $n=19)$, glioma tumor tissues $(n=79)$ and glioma cell lines (U251 and A172). ${ }^{*} P<0.01$ vs. NBTs group. $\mathbf{b}$ Expression levels of ATB in glioma tissues of positively correlated with tumor grades. ${ }^{*} P<0.01$ vs. NBTs group. c Kaplan-Meier analyses of the associations between ATB expression level and overall survival of patients with human glioma (The log-rank test was used to calculate $P$-values). ${ }^{* *} P<0.01$ vs. low ATB expression group. d Relative expression levels of ATB after glioma cells transfected with sh-ATB and sh-control. ${ }^{* *} P<0.01$ vs. sh-control group 
relationship between the expression of ATB, miR-200a and TGF- $\beta 2$ in tissues was analyzed with Pearson's correlation. Survival analysis was performed using the log-rank test in GraphPad Prism 5. Differences were considered significant if $P<0.05$. Corresponding significance levels are indicated in the figures.

\section{Results}

\section{High expression of ATB was correlated with poor outcome of glioma patients}

To define the role of ATB in glioma, using real-time quantitative PCR (RT-QPCR) analysis, we measured ATB expression levels in 79 glioma tissues, 19 normal brain tissues and two glioma cell lines (U251 and A172). ATB expression levels in glioma tissues and cell lines were significantly increased compared with normal brain tissues (Fig. 1a). To determine whether ATB expression was associated with the grade malignancy in gliomas, we examine mRNA expression of ATB. We showed that ATB expression was positively correlated with the pathological grades of glioma (Fig. 1b). We then further assess whether the expression of ATB is correlated with the postoperative survival time of human glioma. The Kaplan-Meier method and log-rank test revealed that high ATB expression level was inversely correlated with glioma patients' overall survival (Fig. 1c).

\section{Knockdown of ATB inhibited glioma cells proliferation, migration, invasion in vitro}

To explore the biological functions of ATB in glioma, we firstly designed an ATB knockdown model using transfection of sh-ATB plasmid in U251 and A172

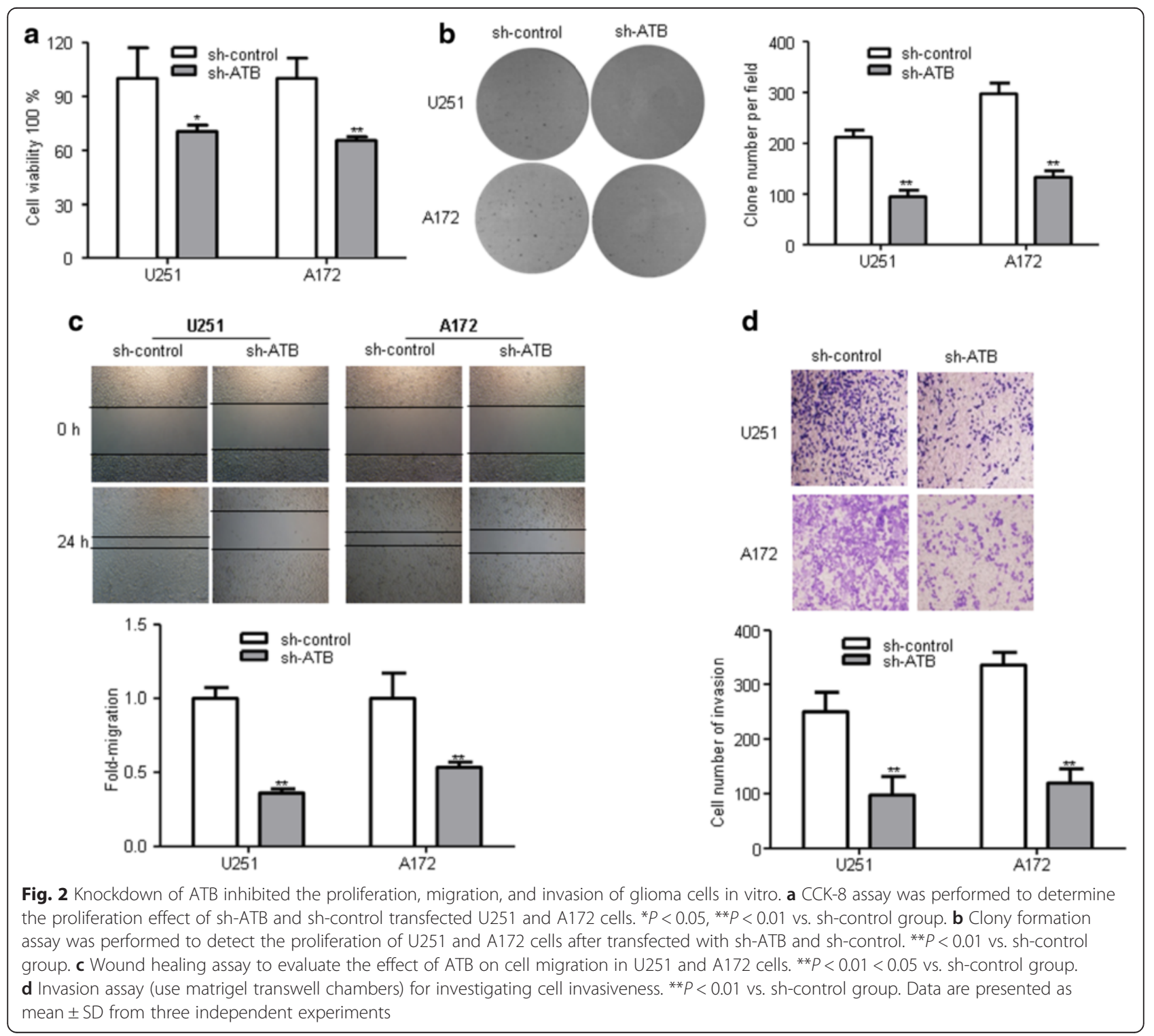


glioma cell lines and its knockdown efficiency was remarkable compared with sh-control (Fig. 1d). CCK-8 assays were performed to detect the impact of ATB knockdown on cell viability of glioma cell lines (U251 and A172). We found that ATB knockdown inhibited cell viability compared to that of cells transfected with sh-control group (Fig. 2a). Furthermore, colony formation assay showed that ATB knockdown exhibited remarkable decreased clone numbers and colony size compared with sh-control group both in U251 and A172 glioma cells (Fig. 2b). To further explore the effect of ATB on glioma cells migration and invasion. Wound healing assay showed that knockdown of ATB impeded the migration in monolayer cultured U251 and A172 glioma cells (Fig. 2c). In addition, numbers of invaded cells were obviously attenuated in the sh-ATB groups compared with sh-control groups (Fig. 2d). Therefore, these results indicated that ATB might act as an oncogene in regulating glioma biological process.

\section{ATB directly targeted miR-200a and inhibited its expression in glioma cells}

Previous studies have demonstrated that lncRNAs could function as a competing endogenous RNAs (ceRNA) by competitively binding miRNAs, such as miR-200a, in hepatocellular carcinoma $[8,13,19]$.

Thus we intended to investigate the potential interaction between ATB and miR-200a in human glioma. As shown Fig. 3a, using QRT-PCR analysis, we found that the expression of miR-200a was lowly expressed in glioma tissues compared with normal brain tissues (NBTs). We further found that miR-200a level in glioma tissues was inversely correlated with pathological grades of glioma (Fig. 3b). We further found that the expression level of ATB was inversely correlated with the expression of miR-200a in 79 human glioma patients (Fig. 3c).

Then, U251 and A172 glioma cells were transfected with miR-200a NC, miR-200a mimics and miR-200a inhibitors (Fig. 3d). To further elucidate whether ATB was regulated by miR-200a, we compared ATB expression levels in $\mathrm{U} 251$ and A172 cells transfected with miR-200a NC or miR-200a mimics and the results showed that overexpression of miR-200a did not reduce the expression of ATB in glioma cells (Fig. 3e). However, knockdown of ATB significantly increased the expression levels of miR-200a compared to the control group (Fig. 3f).

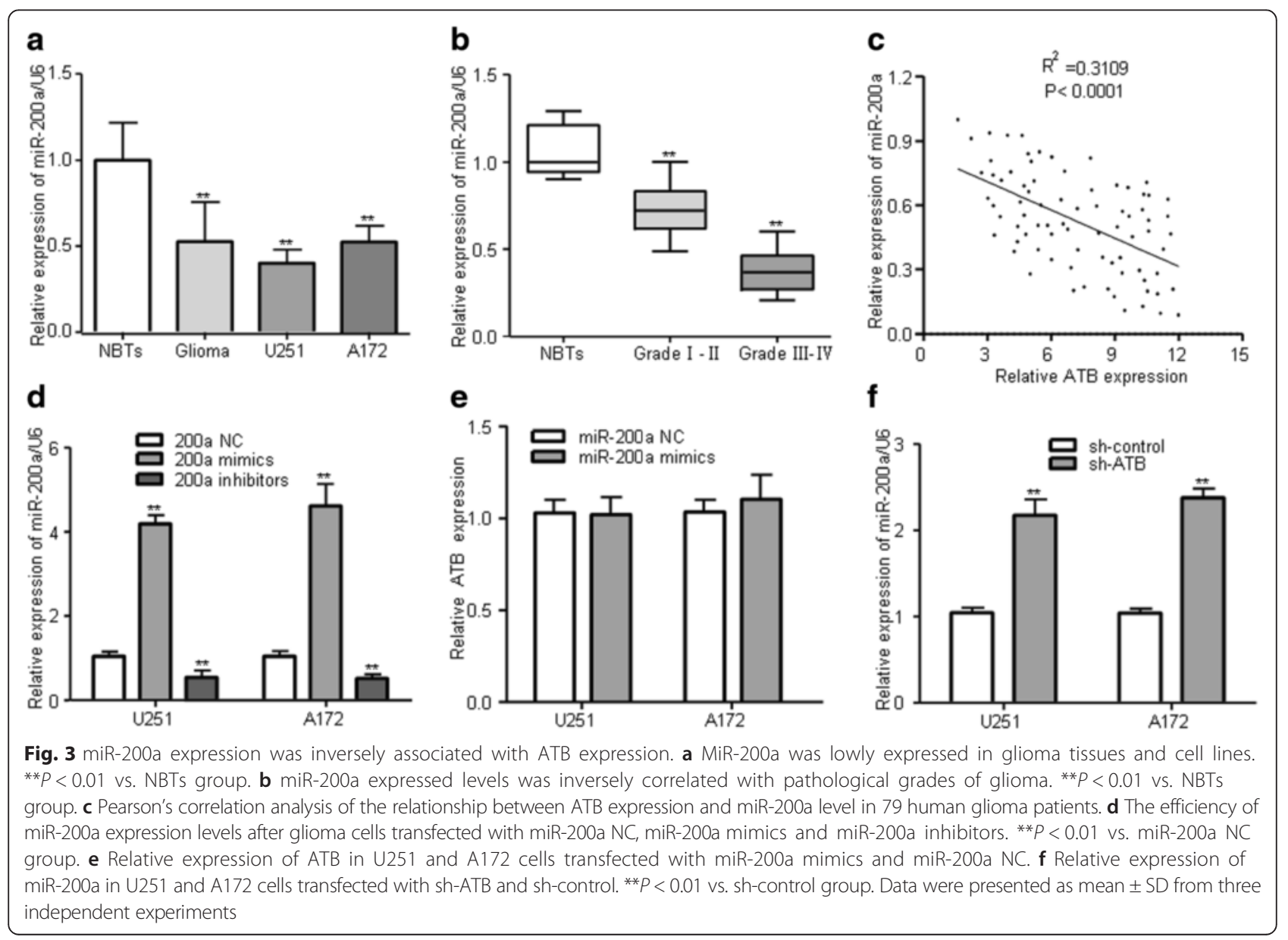


To validate the direct binding between miR-200a and ATB at endogenous levels, we constructed luciferase reporters, which contain wild-type (WT) or mutated (Mut) miR-200a binding sites (Fig. 4a). We found that overexpression of miR-200a could reduce ATB-WT luciferase activity but not affect ATB-Mut luciferase activity compared with miR-200a NC (Fig. 4b-c). The microRNAs are known to bind their targets and cause translational repression and/or RNA degradation in an Ago2-dependent manner. To determine whether ATB was regulated by miR-200a in such a manner, we conducted anti-Ago2 RIP in U251 and A172 cells transiently overexpressing miR-200a. Endogenous ATB pull-down was specifically enriched in miR-200a-transfected cells (Fig. 4d-e), supporting that miR-200 s are bona fide ATB-targeting microRNAs. These data demonstrated that miR-200a bound to ATB but did not induce the degradation of ATB. All these data implied that ATB physically correlated with the miR-200a in glioma cells.

\section{Repression of miR-200a restored the sh-ATB induced inhibitory effects on glioma cells}

In order to investigate whether ATB exerts biological functions through miR-200a, we perform a rescue experiment. The proliferation was reduced in ATB knockdown, while miR-200a inhibitors reversed the reduction of proliferation and miR-200a mimics inhibited the proliferation (Fig. 5a-b). Colony formation assay was used to further assess the proliferation ability. The results showed that ATB knockdown combined with miR200a overexpression resulted in significant reduction of clone numbers and clone size of glioma cells, whereas miR-200a inhibitors recused the clone ability in ATB knockdown glioma cells (Fig. 5c). In addition, ATB knockdown combined with miR-200a overexpression group was strongly reduced invaded cells, and miR-200a inhibitors reversed the invasion of ATB knockdown glioma cells (Fig. $5 \mathrm{~d}$ ). Therefore, these results suggest that ATB acts its tumor-oncogene roles though miR-200a in glioma cells.

\section{ATB regulated TGF- $\beta 2$, a target of miR-200a, in glioma cells}

To investigate whether miR-200a targeted TGF- $\beta 2$ in glioma cells, and TargetScan software predicted miR-200a binding sites in the 3'UTR of human TGF- $\beta 2$ (Fig. 6a). The expression levels of TGF- $\beta 2$ in glioma cells transfected with miR-200a NC, mimics and inhibitors were tested. Overexpression of miR-200a significantly reduced both mRNA and protein expression levels of TGF- $\beta 2$ compared to miR-200a NC while inhibited miR-200a expression exhibited the opposite effects (Fig. 6b-c). The dual-luciferase reporter assay showed that the luciferase activity in the TGF- $\beta 2-W T$ was significantly decreased after transfection with miR-200a mimics compared to miR-200a NC, whereas the luciferase activity in the

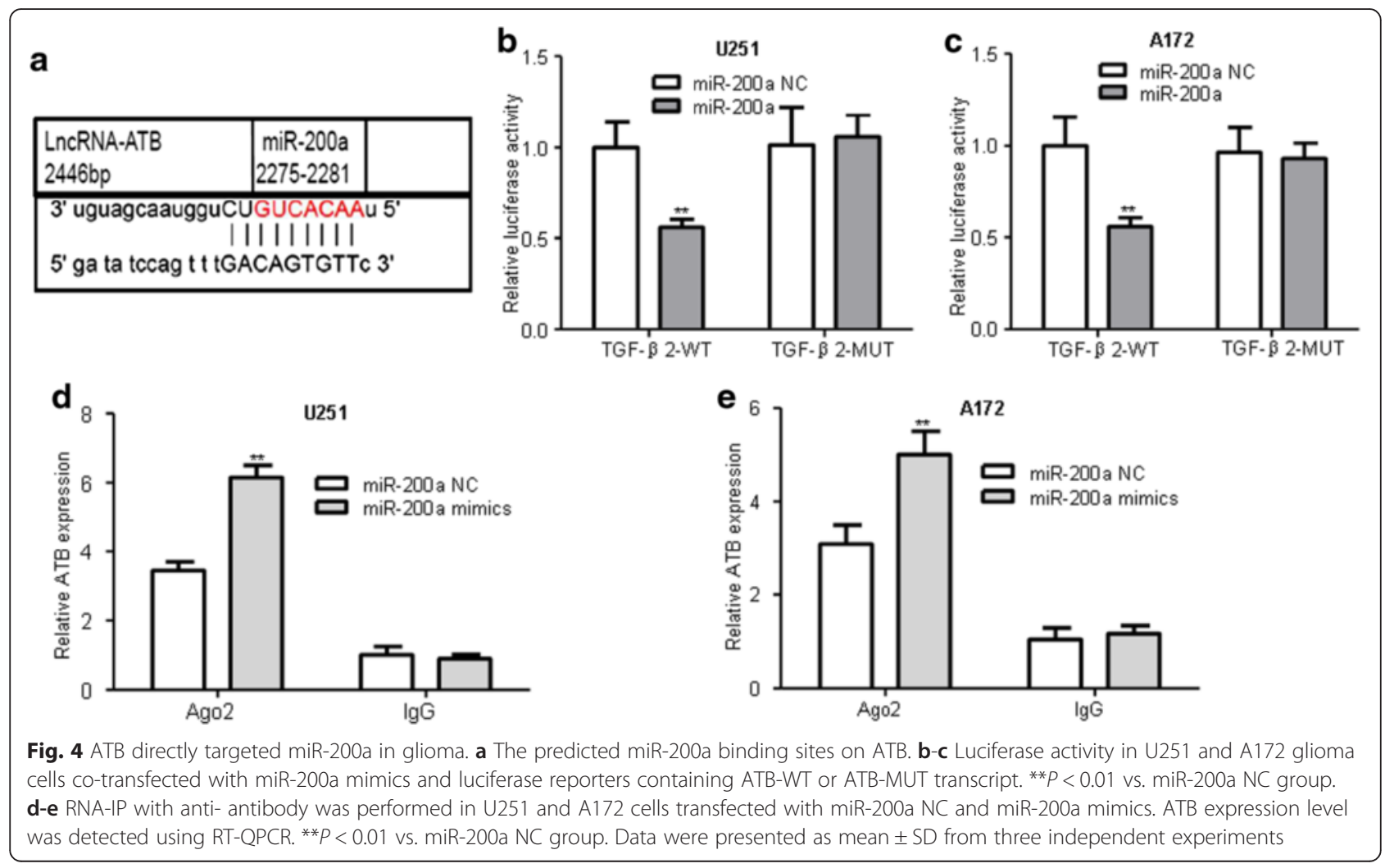




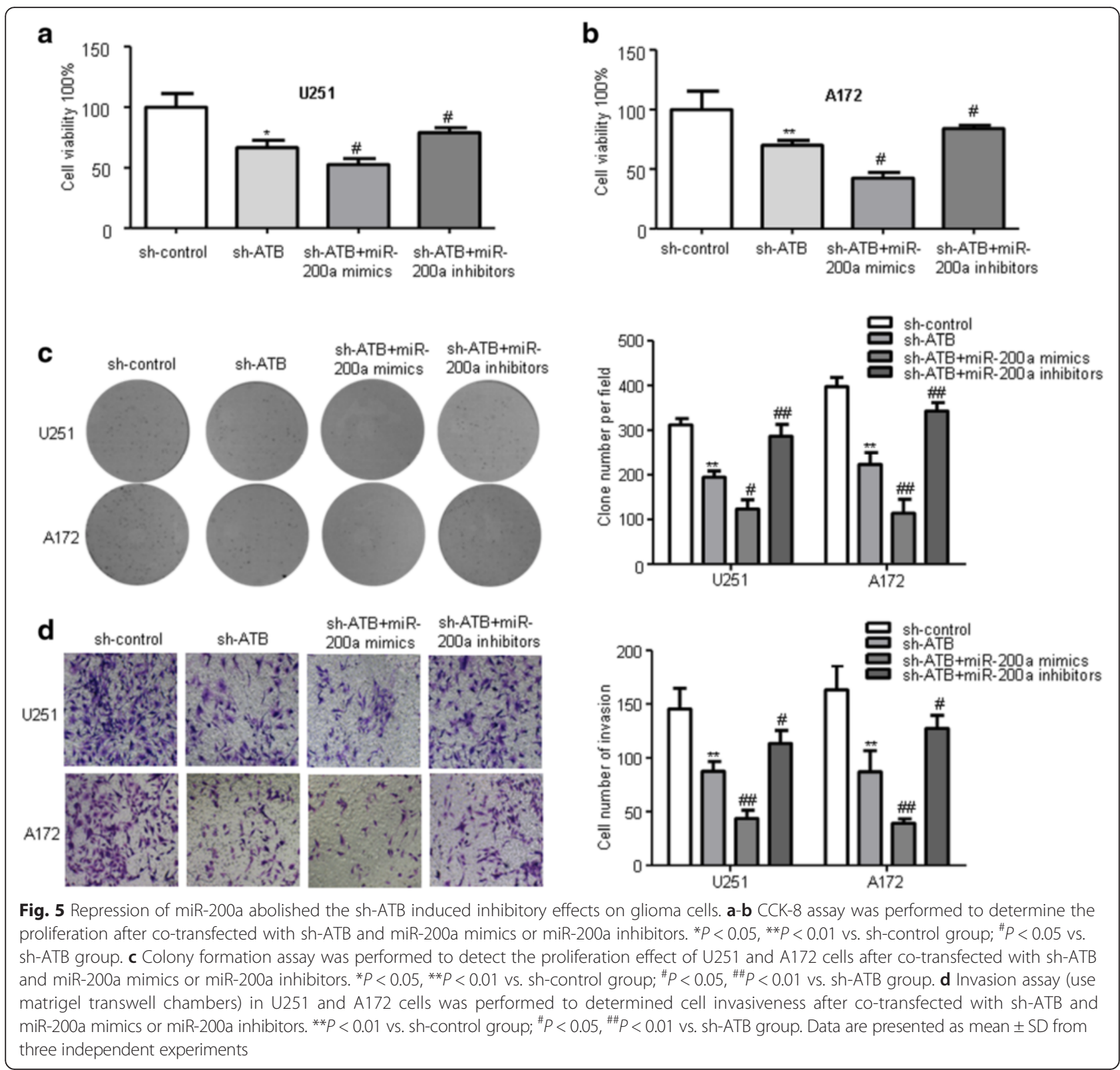

TGF- $32-M U T$ had no change in cells transfected with miR-200a mimics and miR-200a NC group (Fig. 6d-e). These data indicated that miR-200a inhibited TGF- $\beta 2$ expressions in glioma cells by directly targeting the $3^{\prime}$ UTR of oncogene TGF- $\beta 2$.

To explore whether ATB could function as a ceRNA for TGF- $\beta 2$ via modulating miR-200a in glioma, RTQPCR and Western blot assays were performed to detect the mRNA and protein levels of TGF- $\beta 2$ after cells transfected with sh-ATB combined with miR-200a mimics, miR-200a inhibitors. Knockdown of ATB reduced the expression of TGF- $\beta 2$ at mRNA and protein levels, and sh-ATB combined with miR-200a mimics significantly reduced the expression levels of TGF- $\beta 2$, in contrast, miR-200a inhibitors restored the reduction of TGF- $\beta 2$ expression in ATB knockdown glioma cells (Fig. 7a-c). Additionally, we further found that the expression levels of TGF- $\beta 2$ was inversely correlated with the expression of miR-200a and positively associated with ATB in 79 human glioma patients (Fig. 7d-e). These findings suggest that ATB functions as a ceRNA to regulate the expression of TGF- $\beta 2$ by releasing miR-200a in glioma cells.

\section{ATB inhibition significantly suppressed tumor growth in vivo}

To evaluate the functional roles of ATB in vivo, shcontrol/sh-ATB transfected U251 cells were inoculated 
a

\begin{tabular}{lll}
\hline TGF- $\beta 2$ & WT & 5' AGAGCCUUGGUUCAUCAGUGUUA \\
miR-200a & 3' UGUAGCAAUGGUCUGUCACAAU \\
TGF- $\beta 2$ & MUT 5' AGAGCCUUGGUUCAUGUCACAAA
\end{tabular}

C

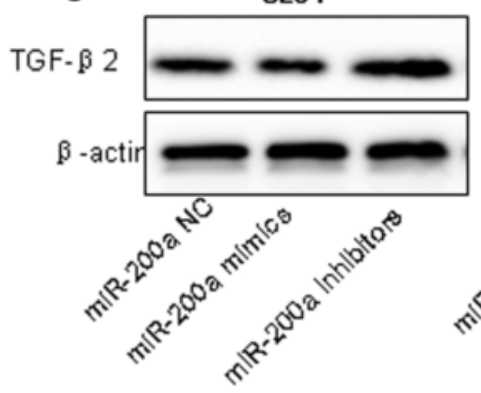

U251

$$
\text { d }
$$

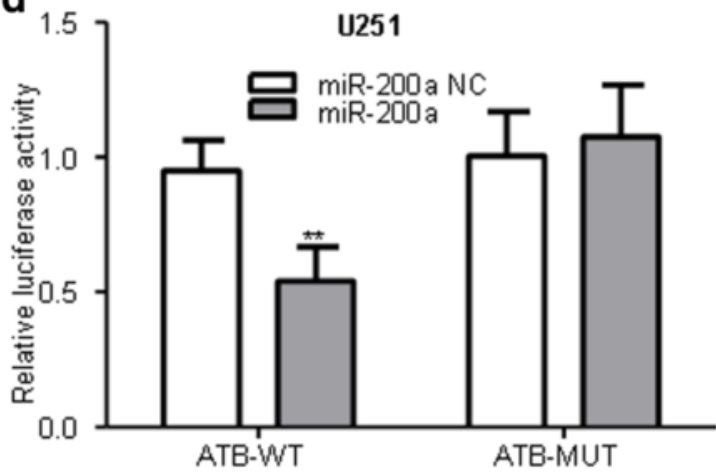

b
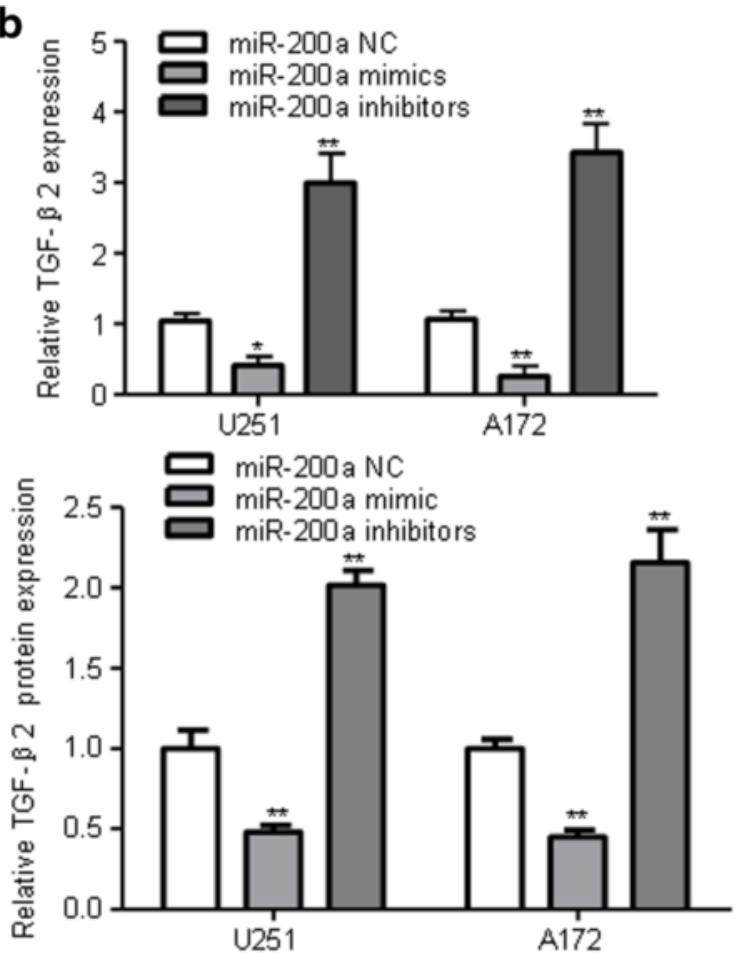

e

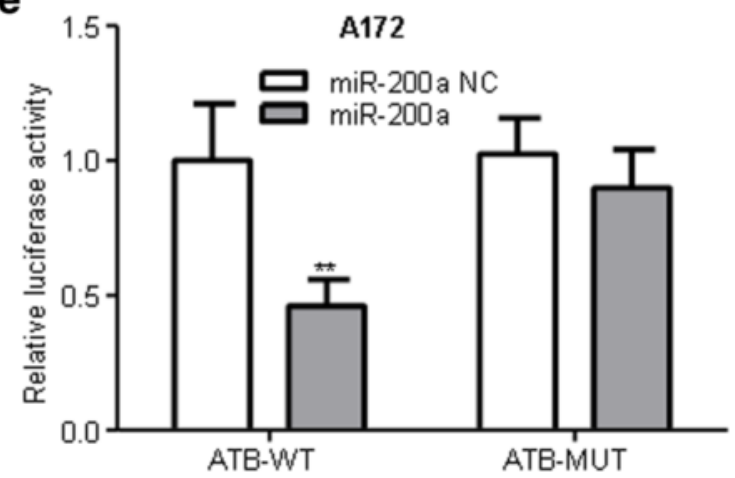

Fig. 6 miR-200a directly targeted the $3^{\prime} U T R$ of oncogene TGF- $\beta 2$ and inhibits its expression in glioma cells. a The predicted of miR-200a binding sites in the 3'-UTR region of TGF- $\beta 2$ (TGF- $\beta 2-3^{\prime}-U T R-W T$ ) and the corresponding mutant sequence (TGF- $\beta 2-3^{\prime}$ UTR-MUT) was shown. b-c Relative expression of TGF- $\beta 2$ mRNA and protein levels in U251 and A172 cells after transfected with miR-200a mimics, miR-200a inhibitors, and miR-200a NC. ${ }^{*} P<0.05,{ }^{* *} P<0.01$ vs. miR-200a NC group. $\mathbf{d}$-e Luciferase activity in U251 and A172 cells co-transfected with miR-200a mimics and luciferase reporters containing TGF- $\beta 2$ wild type (WT) or mutant type (MUT) $3^{\prime}-$-UTR. ${ }^{* *} P<0.01$ vs. miR-200a NC group. Data are presented as mean \pm SD from three independent experiments

into nude mice respectively. Tumor volumes in the sh-ATB group were obviously smaller compared with the sh-control group (Fig. 8a-b). Also, tumor weights in the sh-ATB group were significantly lower than in the shcontrol group (Fig. 8c). Ki-67 staining was performed to measure the proliferation ability in xenografted tumor tissues. As shown in Fig. 8d, the sh-ATB group had fewer proliferative cells than that in the sh-control group.

\section{Discussion}

Nowadays, operation and chemoradiotherapy termed as comprehensive treatments are the main means for the treatment of glioma patients, although it can alleviate patients'suffering and prolong life-span, the prognosis is still poor [20, 21]. Therefore, it is urgent for us to find new effective therapies for glioma patients. Recent studies have suggested that dysregulation of lncRNAs are involved in the tumorigenesis and progression of multiple cancers, including glioma [22, 23]. Consequently, understanding the underlying mechanisms and biological functions of lncRNAs in glioma may give us a new direction for the treatment of glioma patients.

A number of studies have demonstrated that lncRNAs play considerable functional roles in human malignant 


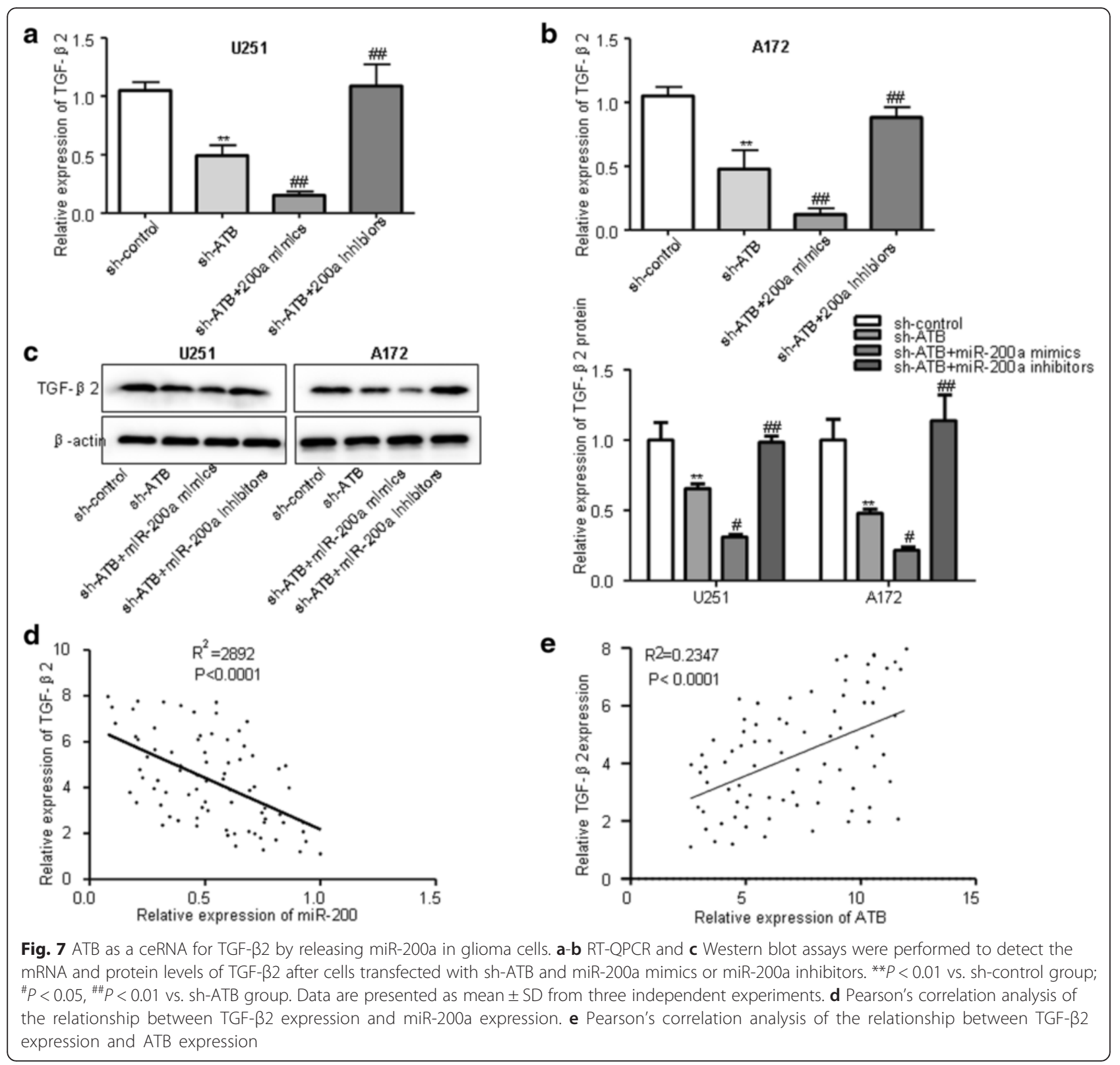

tumor [24-27]. Long non-coding RNA ATB was overexpressed in hepatocellular carcinoma, which promotes HCC cells invasion and tumor growth [13]. Furthermore, ATB is also found as a novel biomarker of lncRNA, indicative of a poor prognosis in gastric cancer and colon cancer patients $[15,28]$. In the present study, our results confirmed that ATB was highly expressed in glioma, and high ATB expression was associated with a poor clinical outcome in glioma patients. Knockdown of ATB suppressed the proliferation, migration and invasion of glioma cells. In addition, the in vivo experiments also supported the above findings, suggesting that ATB exerted as an oncogene in human glioma. Moreover, overexpression of ATB in breast cancer cells promotes the invasiveness, which is consistent with the results of our study [14].

MiRNAs may function as oncogenes and/or tumor suppressors in many cellular processes during cancer initiation and progression [29]. Accumulating evidences have showed that lncRNAs can antagonize miRNA function by competing with miRNAs for binding to shared target mRNAs, and then to silence target mRNAs [30]. Long noncoding RNA ANRIL promotes tumor growth by epigenetically repressing of $\mathrm{miR}-99 \mathrm{a} / \mathrm{miR}-449 \mathrm{a}$ in human gastric cancer [31]. Gas5 suppresses the malignant biological characteristics by down-regulating miR-222 in human glioma [32]. Growing number of present work confirmed the common existence of a widespread 


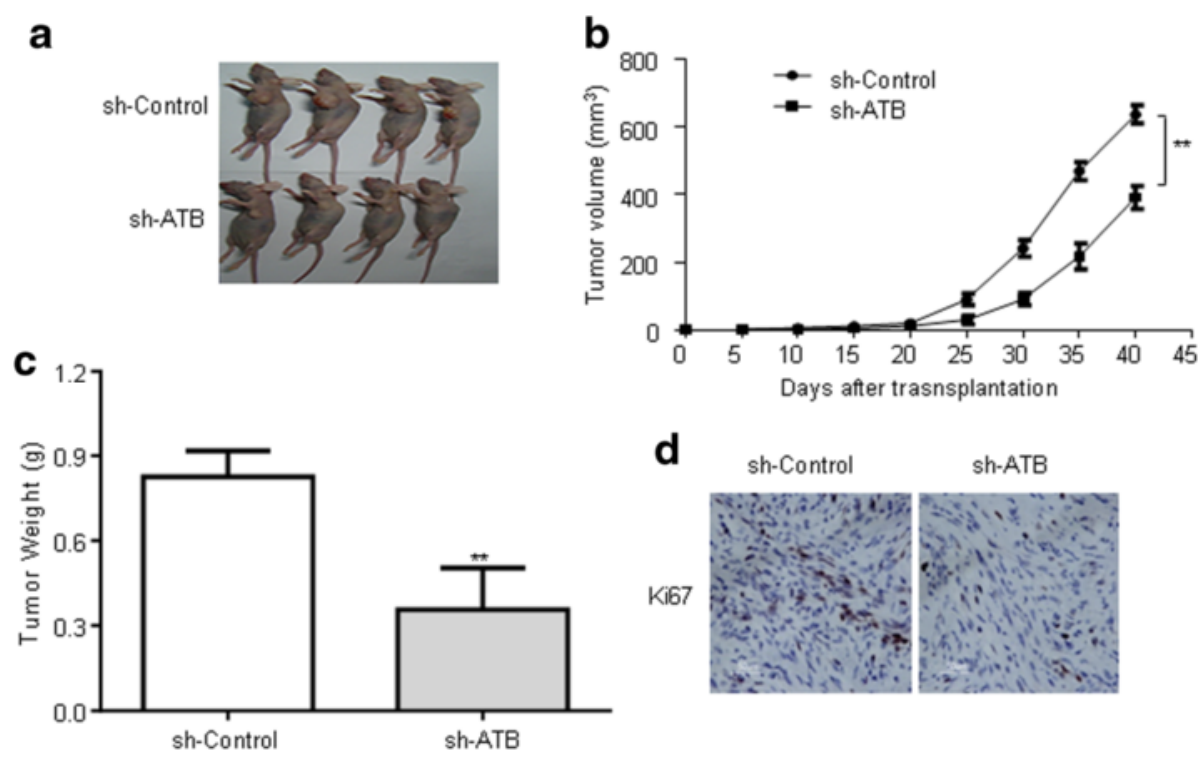

Fig. 8 Knockdown of ATB inhibited U251 cell growth in vivo. a sh-control or sh-ATB was transfected into U251 cells, which were injected in nude mice, respectively. $\mathbf{b}$ Tumor volumes were calculated every 5 days after injection. Bars indicate SD. c Tumor weights are represented as means of tumor weights \pm SD. $\mathbf{d}$ Immunohistochemical $(\mathrm{IHC})$ staining expression of $\mathrm{Ki}-67$ in subcutaneous tumors of mice injected with sh-control or sh-ATB cells. Data are presented as mean \pm s.d. from three independent experiments. ${ }^{*} P<0.01$ vs. sh-control group

interaction network of competitive endogenous RNAs (ceRNAs), in which IncRNAs may exert functions through targeting miRNAs and regulating their function role $[19,33]$. ATB could promote the invasionmetastasis cascade in $\mathrm{HCC}$ by negative regulating of miR-200 family [13]. Previous study showed that downregulation of miR-200a promoted glioma malignancy by up-regulating SIM2-s [18]. Our present study confirmed that miR-200a was significantly decreased in glioma tissues and inversely correlated with ATB. To explore whether ATB has miR-200a binding site in glioma cells, and was confirmed by luciferase reporter assays and RIP. We verified that ATB is directly bound to miR-200a on ATB transcript. RNA-IP assay showed that the expression of ATB immunoprecipitated with in the miR-200a overexpression group was significantly increased. Meanwhile, we found that ATB exerted the function of antitumor by miR-200a in glioma cells. Taken all together, these data strongly suggested that ATB directly targets miR-200a and affects the biological characteristic of glioma cells by negatively regulating miR-200a.

The transforming growth factor- $\beta 2$ (TGF- $\beta 2$ ) belongs to TGF- $\beta$ family, functioned as an oncogene in several cancer types, which promote cancer cells' malignant behaviors [34]. Recent study has reported that miR-200a suppresses renal cell carcinoma development by directly targeting TGF- $\beta 2$ [35] and miR-200a prevents renal fibrogenesis by suppressing of TGF- $\beta 2$ [36]. Consistent with previous studies, we confirmed that miR-200a regulated the expression of TGF- $\beta 2$ via targeting the 3'UTR of TGF- $\beta 2$.
Emerging evidences indicated that lncRNAs play a critical role in a variety of cellar biological processes by acting as a ceRNA or a molecular sponge in modulating the role and functions of miRNAs [30, 37]. These lncRNAs act as a natural miRNA sponge to control endogenous miRNAs by using shared miRNAs responsive elements (MREs) and then modulating the derepression of these miRNAs targets via post-transcriptional regulation [38]. For instance, HOTAIR, a well-known lncRNA could inhibit the expression of FGF1 by regulating miR326 in human glioma [39], and also functioned as a competing endogenous RNA to regulate HER2 expression by sponging miR-331-3p in promoting gastric cancer [40]. To further explore whether TGF- $\beta 2$ is involved in the ATB acts as a ceRNA in regulating the biological characteristics of glioma by modulating miR-200a. We demonstrated that miR-200a reversed the reduction of TGF- $\beta 2$ mediated by ATB knockdown. In addition, we found that TGF- $\beta 2$ expression was negatively correlated with miR-200a, but positively associated with ATB in glioma tissues. These results suggested that ATB functions as a ceRNA via decreasing miR-200a, up-regulating TGF- $\beta 2$ in human glioma.

\section{Conclusion}

In summary, we first reported that ATB was highly expressed in glioma tissues and acted as an oncogene, which serves a key function in regulating glioma malignancy. ATB knockdown suppressed glioma biological characteristics by directly targeting miR-200a and 
negatively regulating its expression in glioma cells. TGF- $\beta 2$, a target oncogene, is directly bound to tumor-suppressor gene miR-200a and is involved in ATB function as a ceRNA for miR-200a in glioma. Therefore, understanding the functional role of ATB in glioma will provide us a novel strategy to find potential therapeutic targets for the treatment of glioma.

\begin{abstract}
Abbreviations
ceRNAs, competing endogenous RNAs; CRNDE, Colorectal neoplasia differentially expressed; DMEM, Dulbecco's modified Eagle's medium; FBS, fetal bovine serum; HCC, Hepatocellular carcinoma; HOTAIR, HOX transcript antisense intergenic RNA; LncRNA-ATB, long noncoding RNA activated by TGF- $\beta$; IncRNAs, long non-coding RNAs; MEG3, Maternally Expressed Gene 3; miRNAs, microRNAs; MREs, miRNAs responsive elements; NBTs, normal brain tissues; ncRNAs, non-coding RNAs; RIP, RNA immunoprecipitation; TGF- $\beta 2$, transforming growth factor- $\beta 2$
\end{abstract}

\section{Acknowledgments}

This project was supported by the National Natural Science Foundation of China (No. 81402078, 81072066), Natural Science Foundation of Anhui Province (No. 1608085MH225, 1508085MH194).

\section{Authors' contributions}

CCM and BEB conceived and designed the experiments. CCM, CW, GNZ and XZ performed the experiments and drafted the manuscript. HLW, GZ and BZ contributed to the design of this study and helped to draft the manuscript. BZ supervised the whole work and revised the manuscript. All authors read and approved the manuscript.

\section{Competing interests}

The authors declare that they have no competing interests.

Received: 15 March 2016 Accepted: 30 May 2016

Published online: 06 June 2016

\section{References}

1. Louis DN, Ohgaki H, Wiestler OD, Cavenee WK, Burger PC, Jouvet A, et al. The 2007 WHO classification of tumours of the central nervous system. Acta Neuropathol. 2007;114:97-109.

2. Wen PY, Kesari S. Malignant gliomas in adults. N Engl J Med. 2008;359:492-507.

3. Carninci P, Kasukawa T, Katayama S, Gough J, Frith MC, Maeda N, et al. The transcriptional landscape of the mammalian genome. Science. 2005;309:1559-63.

4. Krzyzanowski PM, Muro EM, Andrade-Navarro Wiley MA. Computational approaches to discovering noncoding RNA. Interdiscip Rev RNA. 2012;3:567-79.

5. Rinn JL, Chang Annu HY. Genome regulation by long noncoding RNAs. Rev Biochem. 2012;81:145-66.

6. Batista PJ, Chang HY. Cellular address codes in development and disease. Cell. 2013;152:1298-307.

7. Geisler S, Coller Nat J. RNA in unexpected places: long non-coding RNA functions in diverse cellular contexts. Rev Mol Cell Biol. 2013;14:699-712.

8. Cesana M, Cacchiarelli D, Legnini I, Santini T, Sthandier O, Chinappi M, et al. A long noncoding RNA controls muscle differentiation by functioning as a competing endogenous RNA. Cell. 2011;147:358-69.

9. Rinn JL, Kertesz M, Wang JK, Squazzo SL, Xu X, Brugmann SA, et al. Functional demarcation of active and silent chromatin domains in human HOX loci by noncoding RNAs. Cell. 2007;129:1311-23.

10. Gupta RA, Shah N, Wang KC, Kim J, Horlings HM, Wong DJ, et al. Long non-coding RNA HOTAIR reprograms chromatin state to promote cancer metastasis. Nature. 2010;464:1071-6.

11. Graham LD, Pedersen SK, Brown GS, Ho T, Kassir Z, Moynihan AT, et al. Colorectal Neoplasia Differentially Expressed (CRNDE), a Novel Gene with Elevated Expression in Colorectal Adenomas and Adenocarcinomas. Genes Cancer. 2011:2:829-40

12. Li J, Bian EB, He XJ, Ma CC, Zong G, Wang HL, et al. Epigenetic repression of long non-coding RNA MEG3 mediated by DNMT1 represses the p53 pathway in gliomas. Int J Oncol. 2016;48:723-33.
13. Yuan JH, Yang F, Wang F, Ma JZ, Guo YJ, Tao QF, et al. A long noncoding RNA activated by TGF-beta promotes the invasion-metastasis cascade in hepatocellular carcinoma. Cancer Cell. 2014;25:666-81.

14. Shi SJ, Wang LJ, Yu B, Li YH, Jin Y, Bai XZ. LncRNA-ATB promotes trastuzumab resistance and invasion-metastasis cascade in breast cancer. Oncotarget. 2015;6:11652-63.

15. Yue B, Qiu S, Zhao S, Liu C, Zhang D, Yu F, et al. LncRNA-ATB mediated Ecadherin repression promotes the progression of colon cancer and predicts poor prognosis. J Gastroenterol Hepatol. 2015;3:595-603.

16. Bartel DP. Genomics, biogenesis, mechanism, and function. Cell. 2004;116:281-97.

17. Tanaka K, Kawano M, Itonaga I, Iwasaki T, Miyazaki M, S Ikeda S, et al. Tumor suppressive microRNA-138 inhibits metastatic potential via the targeting of focal adhesion kinase in Ewing's sarcoma cells. Int J Oncol. 2016;3:1135-44.

18. Su Y, He Q, Deng L, Wang J, Liu Q, Wang D, et al. MiR-200a impairs glioma cell growth, migration, and invasion by targeting SIM2-s. Neuroreport. 2014;25:12-7.

19. Tay Y, Kats L, Salmena L, Weiss D, Tan SM, Ala U, et al. Coding-independent regulation of the tumor suppressor PTEN by competing endogenous mRNAs. Cell. 2011;147:344-57.

20. Jones $C$ and Baker SJ. Unique genetic and epigenetic mechanisms driving paediatric diffuse high-grade glioma. Nat Rev Cancer. 2014;14. doi:10.1038/nrc3811.

21. Brandsma D, Stalpers L, Taal W, Sminia P, van den Bent Lancet MJ. Clinical features, mechanisms, and management of pseudoprogression in malignant gliomas. Oncol. 2008;9:453-61.

22. Shi Y, Wang Y, Luan W, Wang P, Tao T, Zhang J, et al. Long non-coding RNA H19 promotes glioma cell invasion by deriving miR-675. PLoS One. 2014;9:e86295.

23. Wang P, Liu YH, Yao YL, Li Z, Li ZQ, Ma J, et al. Long non-coding RNA CASC2 suppresses malignancy in human gliomas by miR-21. Cell Signal. 2015;27:275-82.

24. Gutschner T, Diederichs S. The hallmarks of cancer: a long non-coding RNA point of view. RNA Biol. 2012;9:703-19.

25. Gibb EA, Vucic EA, Enfield KS, Stewart GL, Lonergan KM, Kennett JY, et al. Human cancer long non-coding RNA transcriptomes. PLoS One. 2011;6:e25915.

26. Meng J, Li P, Zhang Q, Yang Z, Fu S. A four-long non-coding RNA signature in predicting breast cancer survival. J Exp Clin Cancer Res. 2014:33:84.

27. Zhou M, Zhao H, Wang Z, Cheng L, Yang L, Shi H, et al. Identification and validation of potential prognostic IncRNA biomarkers for predicting survival in patients with multiple myeloma. J Exp Clin Cancer Res. 2015:34:102.

28. Saito T, Kurashige J, Nambara S, Komatsu H, Hirata H, Ueda M, et al. A Long Non-coding RNA Activated by Transforming Growth Factor-beta is an Independent Prognostic Marker of Gastric Cancer. Ann Surg Oncol. 2015;22 Suppl 3:915-22.

29. Di Leva G, Croce Trends CM. Roles of small RNAs in tumor formation. Mol Med. 2010;16:257-67.

30. Fejes-Toth K, Sotirova V, Sachidanandam R, Assaf G, Hannon GJ, Kapranov P, et al. Post-transcriptional processing generates a diversity of 5 '-modified long and short RNAs. Nature. 2009;457:1028-32.

31. Zhang EB, Kong R, Yin DD, You LH, Sun M, Han L, et al. Long noncoding RNA ANRIL indicates a poor prognosis of gastric cancer and promotes tumor growth by epigenetically silencing of miR-99a/miR-449a. Oncotarget. 2014:5:2276-92

32. Zhao X, Wang P, Liu J, Zheng J, Liu Y, Chen J, et al. Gas5 Exerts Tumorsuppressive Functions in Human Glioma Cells by Targeting miR-222. Mol Ther. 2015;23:1899-911.

33. Kallen AN, Zhou XB, Xu J, Qiao C, Ma J, Yan L, et al. The imprinted H19 IncRNA antagonizes let-7 microRNAs. Mol Cell. 2013;52:101-12.

34. Ikushima H, Miyazono Nat K. TGFbeta signalling: a complex web in cancer progression. Rev Cancer. 2010;10:415-24.

35. Lu R, Ji Z, Li X, Qin J, Cui G, Chen J, et al. Tumor suppressive microRNA-200a inhibits renal cell carcinoma development by directly targeting TGFB2. Tumour Biol. 2015;36:6691-700.

36. Wang B, Koh P, Winbanks C, Coughlan MT, McClelland A, Watson A, et al. miR-200a Prevents renal fibrogenesis through repression of TGF-beta2 expression. Diabetes. 2011;60:280-7.

37. Karreth FA, Pandolfi Cancer PP. ceRNA cross-talk in cancer: when ce-bling rivalries go awry. Discov. 2013;3:1113-21.

38. Sen R, Ghosal S, Das S, Balti S, Chakrabarti J. Competing endogenous RNA: the key to posttranscriptional regulation. Sci World J. 2014;2014:896206. 
39. Ke J, Yao YL, Zheng J, Wang P, Liu YH, Ma J, et al. Knockdown of long noncoding RNA HOTAIR inhibits malignant biological behaviors of human glioma cells via modulation of miR-326. Oncotarget. 2015;6:21934-49.

40. Liu XH, Sun M, Nie FQ, Ge YB, Zhang EB, Yin DD, et al. Lnc RNA HOTAIR

functions as a competing endogenous RNA to regulate HER2 expression by sponging miR-331-3p in gastric cancer. Mol Cancer. 2014;13:92.

Submit your next manuscript to BioMed Central and we will help you at every step:

- We accept pre-submission inquiries

- Our selector tool helps you to find the most relevant journal

- We provide round the clock customer support

- Convenient online submission

- Thorough peer review

- Inclusion in PubMed and all major indexing services

- Maximum visibility for your research

Submit your manuscript at www.biomedcentral.com/submit 